\title{
Design and Application of Real-Time Visual Attention Model for the Exploration of 3D Virtual Environments
}

\author{
Sébastien Hillaire, Anatole Lécuyer, Tony Regia-Corte, \\ Rémi Cozot, Jérome Royan, and Gaspard Breton,
}

\begin{abstract}
This paper studies the design and application of a novel visual attention model meant to compute users gaze position automatically, i.e. without using a gaze-tracking system. The model we propose is specifically designed for real-time first-person exploration of 3D virtual environments. It is the first model adapted to this context which can compute, in real-time, a continuous gaze point position instead of a set of 3D objects potentially observed by the user. To do so, contrary to previous models which use a mesh-based representation of visual objects, we introduce a representation based on surface-elements. Our model also simulates visual reflexes and the cognitive process which takes place in the brain such as the gaze behavior associated to first-person navigation in the virtual environment. Our visual attention model combines the bottom-up and top-down components to compute a continuous gaze point position on screen that hopefully matches the user's one. We conducted an experiment to study and compare the performance of our method with a state-of-the-art approach. Our results are found significantly better with more than $100 \%$ of accuracy gained. This suggests that computing in real-time a gaze point in a 3D virtual environment is possible and is a valid approach as compared to object-based approaches. Finally, we expose different applications of our model when exploring virtual environments. We present different algorithms which can improve or adapt the visual feedback of virtual environments based on gaze information. We first propose a level-of-detail approach that heavily relies on multipletexture sampling. We show that it is possible to use the gaze information of our visual attention model to increase visual quality where the user is looking, while maintaining a high refresh rate. Second, we introduce the use of visual attention model in three visual effects inspired from the human visual system namely: depth-of-field blur, camera motions, and dynamic luminance. All these effects are computed based on simulated user's gaze, and are meant to improve user's sensations in future virtual reality applications.
\end{abstract}

Index Terms - visual attention model, first person exploration, gaze tracking, visual effects, level of detail.

\section{INTRODUCTION}

$\mathrm{T}$ HE gaze point is the point a user is looking at. In Virtual Environments (VE), knowing the gaze point position can give developers several advantages to efficiently display a high quality virtual scene. Applications can take advantage of this feature to better distribute available computational resources to efficiently render a virtual scene [1] or to simulate natural effects occurring in human vision improving users' perception of the VE [2].

A straightforward way to compute user's gaze point position on a screen is to use a gaze tracking system [3]. Since their creation in the late 19th century, before the computer existed, these systems have been considerably enhanced [3]. The interest in these systems has grown thanks to their usefulness in several domains: human studies in psychology to VR systems, aid for people with disabilities or graphic

- M. Hillaire is with Orange Labs and INRIA/IRISA. E-mail: shillair@irisa.fr

- M. Lécuyer and M. Regia-Corte are with INRIA.

- M. Cozot is with INRIA and the University of Rennes 1.

- M. Royan and M. Breton are with Orange Labs. rendering. However, accurate gaze tracking systems are still expensive and can only be accessed by a limited number of researchers or companies.

Another way to compute the gaze point is to use a visual attention model simulating human attention. A lot of research efforts have been dedicated to the evaluation of human attention when looking at pictures [4] or video [5] and its simulation using visual attention models. However, these models have not yet been studied nor adapted to the context of first person exploration of VE. To the best of our knowledge, only the model proposed by Lee et al. [6] is adapted to this context but it can only compute attention per objects.

In this paper, we propose a novel visual attention model adapted to the context of real-time firstperson exploration of VEs. It is composed of new, and improved, components and data representations. We also report on the wide application possibilities the gaze point offers and present several methods to take advantages of it. Our main contributions are:

- A visual attention model specifically designed for real-time exploration of 3D VEs which can compute a continuous 2D gaze point position. This model notably introduces: 
- A novel representation of visual objects based on surface-elements (surfels). We will show that this representation has several advantages over the mesh-based representation for visual attention models;

- The use of a novel component simulating the gaze behavior of users walking and turning in VEs [7], implemented for the first time in a real-time visual attention model;

- An extended evaluation of our model as compared to a state-of-the-art approach in various virtual environments;

- A presentation of methods taking advantage of the gaze feature in order to apply level-of-detail and perceptually inspired visual effects.

In the remainder of this paper, we will describe related work concerning human visual attention and visual attention models. Then, we will detail the novel visual attention model we propose. We will report on an experiment conducted to evaluate the efficiency of our model as compared to a state-of-the-art model. Last, we will discuss efficiency and usability of the proposed model in VR applications. Finally, we will expose several visual effect adapted in real-time to the gaze point position of the user for the purpose of level-of-detail and visual feedback improvement.

\section{Related Work}

Visual attention represents the capacity of a human to focus on a visual object. It is well known that human visual attention is composed of two components [5]: bottom-up and top-down components.

The bottom-up component represents the visual reflexes of the human visual system. Due to the structure of our brain and the fact that we only accurately perceive the environment within 2 degrees of the visual field [8], the human visual system does not have the capabilities to analyze a whole scene in parallel. Actually, the human visual system can only perceive primitive features in parallel [9] in order to detect highly contrasted areas. According to the feature integration theory [9], all this contrast information is combined all together to finally identify salient areas in the visual field. Then, the human visual system uses a sequential visual search to quickly analyze the scene [9]. For example, when someone first looks at a scene, his/her gaze is first unconsciously attracted by visually salient areas to rapidly perceive the most important parts of the scene [4]. Several visually salient features have been identified in previous research [9][4]: red/green and blue/yellow antagonistic colors, intensities, orientations, etc. Inspired by the feature integration theory [9], bottom-up visual attention models have been developed to compute a saliency map from an image [4]. The saliency value of each pixel of the saliency map represents its attractiveness, i.e. the higher the saliency of an area, the more a human is likely to look at this area. Other features have been progressively added in the computation of saliency maps such as flickering [5], depth [6] or motion [5].

Moreover, visual attention is not only controlled by reflexes resulting from visual stimuli, but also by the cognitive process that takes place in the brain, i.e. the top-down component. It is involved in the strategies we use to analyze a scene and is highly dependent on the knowledge, memory and task. For example, Yarbus [10] has shown that the way people look at pictures strongly depends on the task they have to achieve. Furthermore, the top-down component is subject to the habituation phenomenon [11], i.e. objects become familiar over time, and we become oblivious to them. Several models have been proposed to simulate the multiple top-down components using task-map [8], habituation [11], memory [12] as well as spatio-temporal contexts [6].

Bottom-up only models[4][5] have been reported as good predictors as they were able to predict a non-negligible fraction of human gaze targets, i.e. there is a strong correlation between gaze positions and salient areas in a saliency map. However, in a game scenario, Sundstedt et al. [13] have shown that a saliency map alone is not sufficient to efficiently compute user's gaze. Even without an explicit task, users will automatically assume a task by themselves. As suggested by [5], it seems that a saliency map only suggests a set of potential gaze locations, and that another higher level component, i.e. top-down, may choose a gaze position in this set. As a result, it seems necessary for a visual attention model to simulate both bottom-up and top-down components [13].

Sears et al. [14] suggested that human visual attention could be based on objects in their Multiple Object Tracking (MOT) theory. Following this theory, each object has an attention priority value that is assigned in a stimulus-driven manner. A set of 3 to 5 objects are then indexed based on this value. This set of indexed objects can then be attended rapidly and before other objects in the visual field.

Surprisingly, few researches have been dedicated to the use of visual attention models for real-time attention or gaze prediction during exploration of 3D VEs. To our best knowledge, only the visual attention model based on the MOT theory proposed by Lee et al. [6] has been specifically designed for this aim. As described in the MOT theory, it does not compute a gaze point position on the screen but returns the object, or set of objects, that could potentially receive more attention from the user. It is able to predict the object gazed by the user $48 \%$ of the time during free navigation and $62 \%$ of the time during a research task involving navigation in static and dynamic VE. However, this mesh-based discretization might be considered as a limitation as it is not possible to know precisely where the user is looking at on a 


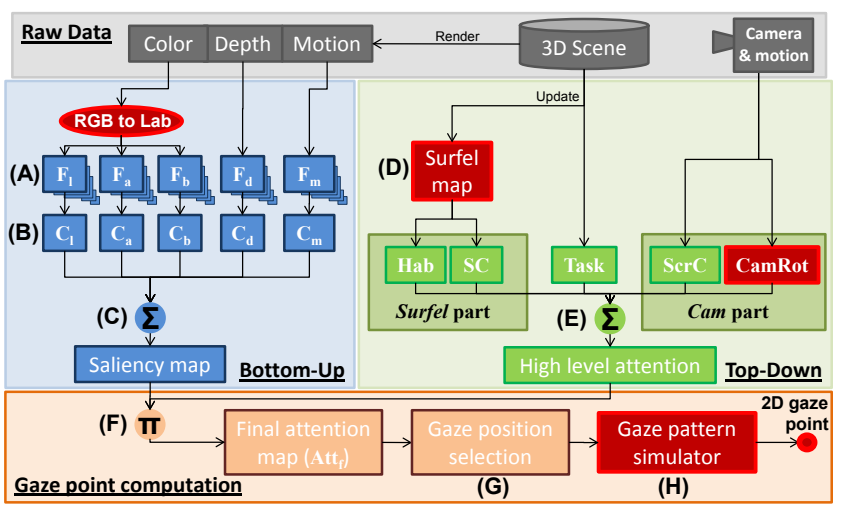

Fig. 1. Overview of our visual attention model architecture. A) feature maps, B) conspicuity maps, C) bottomup attention (saliency map), D) update of per-surfel data, E) top-down attention, F) computation of final attention on screen, G) computation of the possible next gaze position and $\mathrm{H}$ ) the gaze pattern simulator computing the final gaze position on screen. Red color emphasizes the novel parts of our visual attention model compared to existing techniques.

particular object, e.g. is he looking at the corner or middle of a wall? This can be viewed as a coarse approximation, especially for large objects, and it is important to compute a $2 \mathrm{D}$ point when applying gazebased methods such as proposed in [1][2]. As future work, Lee et al. [6] suggested to take into account the novelty of objects and other adapted top-down components. To this aim, Hillaire et al. [7] have studied user's gaze behavior when walking and turning in VEs. They have proposed a new model taking into account first-person navigation in order to compute an attentional weight on the whole screen based on the current rotation velocity of the camera.

\section{A NOVEL VISUAL ATTENTION MODEL FOR REAL-TIME EXPLORATION OF VIRTUAL ENVI- RONMENTS}

This section describes the complete computational visual attention model we propose. This model is able to estimate in real-time a gaze position that hopefully matches user's gaze without the need of physical devices such as web-cam or expensive gaze tracker. In the following subsections, we will describe the computation of both the bottom-up then top-down component. Finally, we will present our method to combine these two components in order to estimate a continuous $2 \mathrm{D}$ gaze position on the screen.

\subsection{Computation of the bottom-up component}

The bottom-up component of our model computes a pixel-level saliency map using several visual features: intensity [4], antagonistic colors [4], depth [11] and motion [5].

\subsubsection{Computation of feature maps}

The starting point to compute a saliency map is to compute a feature map for each visual features (Figure 1-A):

- Antagonistic colors and luminance: Originally, Itti et al. [4] used red/green and blue/yellow antagonistic colors as well as intensities. In their model, antagonistic colors were computed using simple operation on RGB components. In our case, we propose to use the perceptual Lab color space which takes into account human perception [15]. Moreover, this color space has the advantage of directly encoding red/green and blue/yellow antagonistic colors as well as intensity, respectively the $\mathrm{a}, \mathrm{b}$ and $\mathrm{L}$ components. They correspond to $F_{a}, F_{b}$ and $F_{l}$ feature maps in Figure 1.

- Depth: We propose to use a depth map as proposed in [11][6]. The value $F_{d}(p)$ for each pixel $p$ of the depth feature map $F_{d}$ is computed using Equation 1 with $z(p)$ being the linear depth of pixel $p, z_{\text {near }}$ and $z_{f a r}$ the distances of the near and far clip planes.

$$
F_{d}(p)=\frac{z(p)-z_{\text {near }}}{z_{\text {far }}-z_{\text {near }}}
$$

- Motion: Our model also takes into account visible motion on the screen. Lee et al. [6] proposed to approximate this feature using the motion of a single point of each visual object in world space. However this method does not take into account animated objects, e.g. an avatar moving only the hand. The motion feature $F_{m}(p)$ of each pixel $p$ of the motion feature map $F_{m}$ is computed using Equation 2 with $v(p)$ being the world space motion projected on the screen and $t$ the time elapsed since last frame.

$$
F_{m}(p)=\frac{\|v(p)\|}{t}
$$

\subsubsection{Computation of conspicuity maps}

Before computing the saliency map, the feature maps need to be converted into conspicuity maps using the multi-scale Center-Surround difference operator [4] simulating the response of brain neurons which receive stimuli from the visual receptive fields. Instead of a dyadic Gaussian feature map pyramid, we use an approximation consisting of using the fast hardware mipmap pyramid generation of Graphics Processing Units (GPU) (see [6]). The conspicuity maps, i.e. $C_{l}$, $C_{a}, C_{b}, C_{d}$ and $C_{m}$ in Figure 1-B, are computed using Equation 3 with $i$ and $i+j$ being mipmap pyramid levels. The level $i$ is a fine level and $i+j$ a coarser level of the pyramid.

$$
\forall x \in\{l, a, b, d, m\}, C_{x}=\frac{1}{6} \sum_{i=0}^{2} \sum_{j=3}^{4}\left|F_{x}^{i}-F_{x}^{i+j}\right|
$$

Finally, the conspicuity maps are normalized using the $\mathcal{N}$ operator as described by Itti et al. [4] where we replace the mean of local maxima by the mean of all values in the conspicuity map for the sake of performance. 


\subsubsection{Computation of the final saliency map}

The final saliency map can be generated using the conspicuity maps computed in the previous step (Figure 1-C). It is the result of a linear combination of each conspicuity map using Equation 4 . Finally, the saliency map $S$ is normalized in order to have its values mapped into the range $[0,1]$.

$$
S=\frac{1}{5} \times \sum_{x \in\{l, a, b, d, m\}} C_{x}
$$

\subsection{Computation of the top-down component}

The top-down component of our model consists in simulating the cognitive processes that take place in the brain. We first propose a novel representation of visual objects based on surfel (instead of a coarse mesh-based representation) to compute spatial context [6] and habituation [11] components. Our model relies also on screen-space weights to take into account the observed gaze behavior of human navigating in VEs using a first-person view.

\subsubsection{Surfel-based representation of visual objects}

Previous models for 3D exploration of VEs use a representation of visual objects based on meshes [6]. This can be seen as a limitation as it is not possible to differentiate sub-parts of an object. Using this representation, it becomes indeed impossible to know if the user is gazing at the head or leg of an avatar. Moreover, large walls are problematic. Indeed, the wall can be identified as an attended object but it is not possible to differentiate between cases when the user looks at the middle or at a corner of the wall.

A possible solution could be to cut large objects in several parts. However, it is not always possible to easily modify existing assets (3D models). Also, for the sake of performance, subdividing a mesh in several sub-meshes is risky, i.e. too many triangles might impair rendering performance. Furthermore, a visual object could be embedded in the object texture, e.g. bullet holes on a wall.

In this paper, we propose to solve this issue by using a discretization of polygonal surfaces into smaller elements having the same world space size: surface elements also known as surfel. Surfel are well-kown and they are used as a lightmap encoding the irradiance per surface elements [16]. Our visual attention model requires a surfel map to be built for each mesh of every potentially visible object in the VE. In our model, a surfel map virtually subdivides a mesh into several pieces (surfels) and stores them in a texture, thus not involving geometry subdivisions. A surfel is defined by its previous and current positions in world space. Concerning static meshes, the surfel map only contains the current position as visible in Figure 2. Also, for the sake of performance, the surfel map of dynamic objects is updated only when they move.

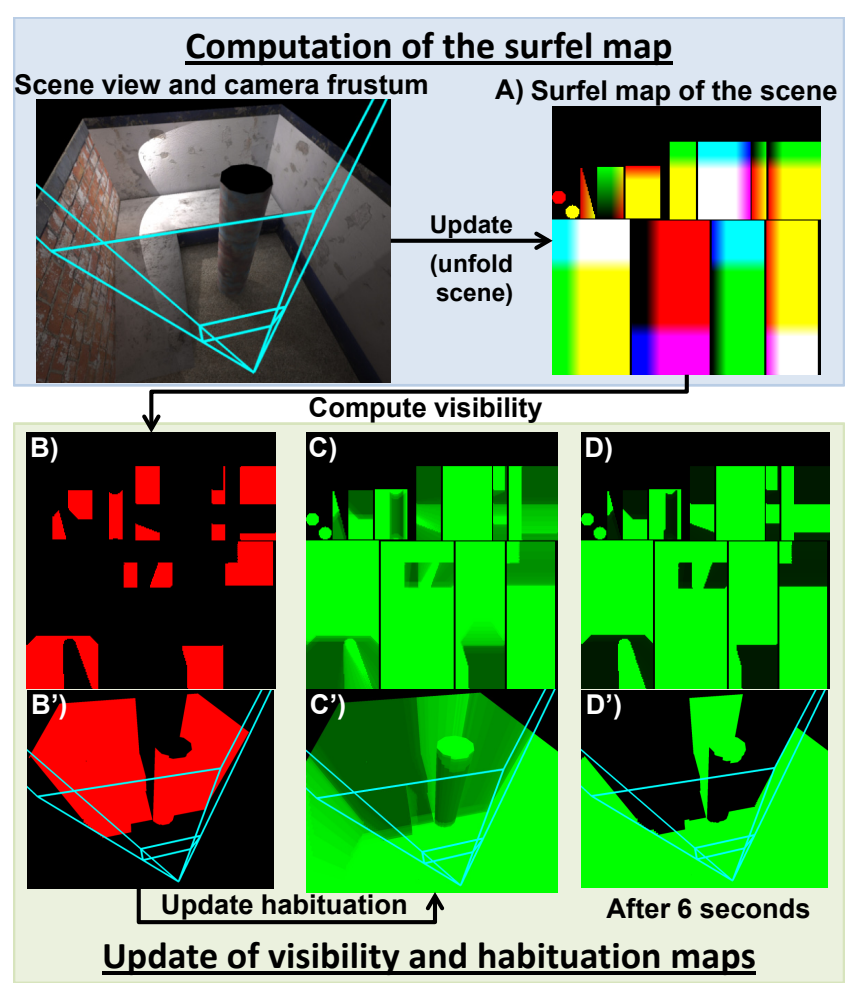

Fig. 2. Computation of the surfel map, and update of visibility and habituation maps. A) the surfel map containing world space surfel position (XYZ into RGB) B) surfel visibility (red=visible), C) surfel habituation (the greener the surfel, the less habituated the viewer is to it) and D) surfel habituation after waiting 6 seconds. B', C' and D' are views of the surfel map texture mapped on the scene.

There are several advantages in using a surfel representation of visual objects. First, the visual object representation is no longer dependent on the way the scene has been modeled: each surfel is represented as a small square patch having the same size in world space. Second, thanks to this uniform world space size, we obtain a uniform distribution of visual objects. Last, each surfel can be easily represented and stored in a texture (see section 3.2.2). Thus, their update and use for visual attention estimation can take advantage of the computational power of modern graphic hardware by processing all surfels in parallel.

\subsubsection{Generating the surfel maps}

To generate the surfel map, which is in fact a texture, we first need texture coordinates for each mesh triangle that will map each triangle to its corresponding $2 \mathrm{D}$ area in the texture. This corresponds to unfolding triangles of the 3D meshes in the 2D texture. These texture coordinates must respect two constraints: (1) overlapping triangles are forbidden and (2) a triangle must at least contain the center of a texture element (texel) of the surfel map to result in a surfel data that can be effectively used later. 3D modeling softwares 
such as Maya or 3D Studio Max already propose such a feature. This process is already used in several applications for the purpose of light-mapping [16].

To fill the surfel map with data, we simply render the 3D meshes into the texture. But, instead of applying a 3D projection, we project the meshes' triangles in $2 \mathrm{D}$ using the texture coordinates as positions. For each texel, the final 3D coordinates in world space are written in the surfel map according to each model transformation matrix (Figure 1-D). The habituation map is created when the application starts. At runtime, few areas of the habituation map are updated for dynamic objects and only when they move. Then, all per-surfel computations required by the visual attention simulation are done in the surfel map using the highly parallel computation power of graphic hardware.

After preliminary testing, we have set the world space size of surfels to $20 \mathrm{~cm}$. Objects smaller than $20 \mathrm{~cm}$, i.e. small keys in our experiment, are virtually scaled-up. In these cases, we have visually adjusted the scale factor to have each object's surfaces represented by at least one surfel. Each VE presented in Section 4 fit in a single $256 \times 256$ surfel map with the dynamic objects being stored in a second $256 \times 256$ surfel map.

\subsubsection{Computation of per-surfel components}

Our visual attention model computes two attentional components per surfel (or visual object) which are habituation and spatial context:

- Habituation: The habituation component refers to the fact that visual objects become familiar over time [11]. For each surfel, we compute the habituation value for current frame using Equation 5 where $t$ is the elapsed time in milliseconds.

$$
H a b(s)= \begin{cases}H a b_{\text {prev }}(s) * \exp \left(-\frac{t}{h^{-}}\right) & \text {if vis(s)==true } \\ \operatorname{Hab}_{\text {prev }}(s)+h^{+} \times t & \text { otherwise }\end{cases}
$$

Surfel are first initialized with a high interest value. When a surfel $s$ become visible, the habituation is attenuated using an exponential decay [11] with interest for $s$ going under 0.1 in $7 \mathrm{~s}\left(h^{-}=3000\right)$ (see [11] for details). When $s$ is not visible, it is linearly regaining full interest in 20s $\left(h^{+}=20000\right)$. The visibility vis $(s)$ of a surfel $s$ is determined using shadow mapping [17] (Figure 2-A) and take advantage of the already computed depth buffer of the rendered view point in the scene. Thanks to the surfel-based representation of visual objects, the visual attention model habituates itself to visible parts of a wall but not to the parts that are hidden by other objects (Figure 2-B). Then, when the user is moving in the VE, area that are discovered/new have a high interest and are more likely to attract his/her attention.

- Spatial context: Lee et al. [6] have proposed a spatial context component to take into account the spatial behavior of the user. This component modulates the importance of each visual object based on its distance to the user. Visual objects (surfels in our case) too close or too far from the distance of interest become progressively less important. We use the same equation as proposed by Lee et al. [6] except that we have removed the condition on the fact that objects must move toward the camera. This was made in order to avoid discontinuities in the spatial context value (Equation 6-SC $(s)$ ). Since users tend to get close to objects they want to inspect [6], our spatial component also gives more importance to surfels moving toward the camera (Equation 6-SC $C_{\Delta d}(s)$ ). For each surfel $s$, the final spatial context value $S C(s)$ is computed using Equation 6:

$$
\begin{aligned}
& S C(s)=S C_{d}(s) * S C_{\Delta d}(s) \\
& S C_{d}(s)=\frac{d(s)}{C_{1}} \times \exp ^{-\left(\frac{d(s)}{C_{1}}\right)^{2}} \\
& S C_{\Delta d}(s)=\min \left(C_{2} \times \max \left(\frac{\delta d(s)}{\delta t}, 0.0\right), 1.0\right)
\end{aligned}
$$

Where $C_{1}=D / 0.707$ and $D$ is the distance when surfel are considered as more important (see [6]). The other parameters are $t$ the elapsed time since the last frame in seconds, $d(s)$ the distance of the surfel $s$ to the camera and $C_{2}$ is a scaling constant. Afer preliminary testing, we chose $C_{2}=0.87$ (it means that an object moving toward the camera with speed equals to the walking speed of user's avatar will have the highest importance value).

\subsubsection{Computation of statistical screen-space com- ponents}

The gaze behavior of users exploring VEs has been studied in [7][18]. The top-down component of our visual attention model takes into account the observed gaze behavior resulting from the fact that users are navigating in the VE using a first-person view.

- Global screen-space gaze density: It has been shown that during a first-person view game, users tend to look more at the center of the screen [18]. We propose to model this behavior as in [6] using a constant weight $\operatorname{Scr} C(p)=\exp ^{-\operatorname{Dist2Center}(p)}$ applied on the screen where Dist2Center $(p)$ is a function giving the distance of pixel $p$ to the center of the screen (Figure 1-E). Screen coordinates are in the range $[0,1]$ and the middle of the screen is $(0.5,0.5)$.

- Gaze behavior during camera rotations: In our model, we introduce for the first time the real-time attentional component recently proposed in [7]. In this paper, authors have studied user's gaze behavior when turning in VEs. They have proposed a new function able to compute an attentional weight on the whole screen corresponding to the current rotation velocity of the camera. For instance, for a yaw rotation of the camera to the left, a high attention weight is set to pixels on the left of the screen. Our model introduces this function to compute an attention value $\operatorname{CamRot}(p)$ for each pixel $p$ on the screen. 


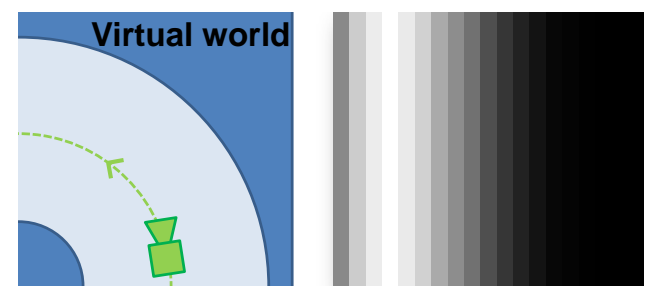

Fig. 3. Attentional weights computed on screen (right) in the case of a left-turn of $100^{\circ} / \mathrm{s}$ (left) in the virtual environment using in real-time the component described in [18] (white pixels correspond to high attention levels).

Using these two screen-space attentional weights, our visual attention model takes into account important gaze behaviors observed during real-time firstperson navigation in VEs.

\subsection{Final screen-space attention and gaze posi- tion computation}

To compute the final 2D gaze position on the screen, the bottom-up and top-down components described previously need first to be combined in a single screen-space attention map.

\subsubsection{Final screen-space attention map}

Previous methods adapted to our context have proposed to compute the user's level of attention for each visual objects in the scene. To do so, the saliency value is modulated with the top-down attention value [6]. Then, 1 to 3 objects having the highest attentional values are considered as the set of potentially gazed visual objects. However, the use of such a model might be problematic notably when the potentially gazed object is very large on the screen. In our model, we remove this constraint by computing a single continuous gaze position on the screen.

To compute the final screen-space attentional map $A t t_{f}$, we first compute the top-down attention value $T D(p)$ for each pixel $p$ on the screen using Equation 7 (Figure 4-B). This is achieved by rendering visible objects from the camera's point of view. In this equation, $\operatorname{Task}(s)$ is a value in the range $[0,1]$ defining surfel relevance for the current task of the user, acting like a semantic weight, $s$ is the position of the surfel in the surfel map, and $p$ is computed using simple texture projection on meshes of $S c r D$ and CamRot textures. In our case, Task $(s)$ is constant for each surfel of a single mesh to reduce memory used but it could also be stored in the surfel map.

$$
\begin{aligned}
T D(p)= & (\operatorname{Hab}(s)+\operatorname{CamRot}(p)+\operatorname{Task}(s)+ \\
& S C(s)+\operatorname{Scr} D(p)) \times 0.2
\end{aligned}
$$

In the last step, the final attention map $A t t_{f}$ (Figure 4-C) is computed from both the top-down (Figure 4-A) and bottom-up (Figure 4-B) components using Equation 8 (Figure 1-F). Finally, the gaze position

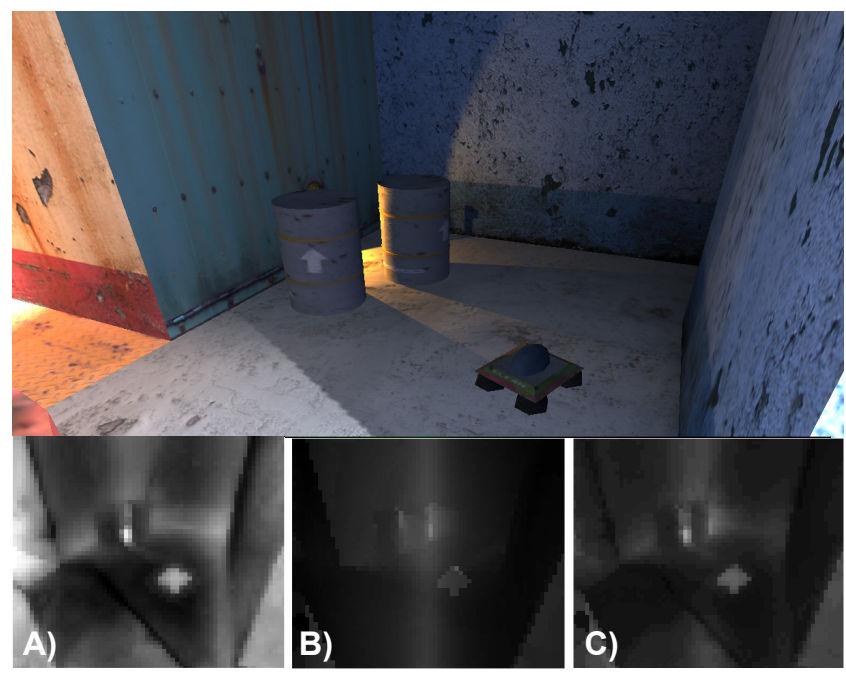

Fig. 4. The saliency map (A), the top-down map (B) and the final attention map (C) computed from a view of a virtual environment.

is selected as the position of the pixel having the highest attention level (Figure 1-G). Then, it is sent to the gaze pattern simulator.

$$
\operatorname{Att}_{f}(p)=\operatorname{vis}(s) \times S(p) \times T D(p)
$$

\subsubsection{Gaze pattern simulator}

We have added a gaze pattern simulator in order to process possible gaze position changes and to smooth out the final gaze position (Figure 1-H).

In the human visual system, the duration of eye saccades is from $120 \mathrm{~ms}$ to $300 \mathrm{~ms}$ long depending on the rotation of the eyes [19], and mean fixation duration varies between $200 \mathrm{~ms}$ and $600 \mathrm{~ms}$. We consider a mean frequency of eyes saccades plus fixation of $600 \mathrm{~ms}$. Thus, in order to smooth the final gaze position, we filter the input gaze position with a low pass using a cut-off frequency of $1.67 \mathrm{~Hz}$. This low pass filter allows the simulation of the smooth pursuit phenomenon [19], occurring when eyes are following a smoothly moving visual object, while allowing fast gaze jumps simulating saccades.

\subsection{Implementation details and performance}

To sum up, our visual attention model combines both bottom-up and top-down attention components into a single attention map. Using this attention map, it finally computes a continuous $2 \mathrm{D}$ gaze position which is filtered by the gaze pattern simulator.

Our visual attention model is implemented using OpenGL and GLSL. We have developed our own exporter from Maya which automatically generates the surfel map texture coordinates. The VEs are rendered using our own rendering engine featuring dynamically shadowed point and spot lights together with global illumination baked in a lightmap using Mental 
Ray software. The renderer also features HDR rendering with simple luminance adaptation. This allows us to study the accuracy of our model in several situation, from very simple to game-like VEs.

During the computation of the bottom-up saliency map, features, and conspicuity, maps are stored in two 3-channel textures containing, respectively $\left(F_{l}, F_{a}, F_{b}\right)$ and $\left(F_{d}, F_{m}\right)$. All the textures used to compute the saliency map have a resolution of $256 \times 256$. Our normalization operator $\mathcal{N}$, simplified as compared to [4], needs parameters such as the maximum and mean values contained in the conspicuity maps. To compute these parameters, we do not iteratively read the entire map using the CPU as this would be too expensive. Instead, we compute the maximum and mean by recursively down-sampling the textures by a factor of two until we reach the size of one texel which contains the final desired values. In this algorithm, at each step, and for each pixel of the coarser level, a fragment program computes the maximum and mean values of the four corresponding pixels sampled from the texture computed in the previous step.

The previous and current surfel positions are required to compute the spatial context component. To do so, updating the surfel maps first requires the copy of texture containing current surfel positions into the texture containing old surfel positions. Then, the current surfel texture is updated only for objects that have moved since last frame. Furthermore, we update the surfel texture only every $100 \mathrm{~ms}$ in order to increase overall performance. To update a surfel map, we must bind it as the current render target and this is a costly operation. To avoid multiple render target switches, the surfel maps of dynamic objects are packed in a large texture atlas.

The final step of our visual attention model consists in computing the final gaze position on the screen as the pixel having the highest attentional value. For this aim, the $\operatorname{Att}_{f}(p)$ is a 3-channel texture which stores the final attention level in the red component and pixel positions in the green and blue components. We then use the same recursive down-sampling method, as to compute the normalization operator parameters, but we keep the coordinates of the pixel having the highest attentional value.

The visual attention model we propose needs several input parameters: linear depth, screen space motion and surfel texture coordinate. This could be considered as computationally too expensive. However these raw data are already computed by many existing 3D game engines to add visual effects such as depth-of-field or motion blur as well as atmosphere light scattering. Thus, adding our model to an existing engine should not require too many changes or additional resources.

Our visual attention model can run in real-time thanks to the graphic hardware. On a laptop PC (Intel Core 2 2.5Ghz, nVidia GeForce3700M, 4Gb of RAM),

\begin{tabular}{|c|c|}
\hline Components & Performance \\
\hline Feature & $0.14 \mathrm{~ms}$ \\
\hline Conspicuity & $0.13 \mathrm{~ms}$ \\
\hline Saliency & $0.18 \mathrm{~ms}$ \\
\hline Per-surfel & $0.45 \mathrm{~ms}$ \\
\hline Total & $0.92 \mathrm{~ms}$ \\
\hline
\end{tabular}

TABLE 1

Computation time in milliseconds for each step of the visual attention simulation.

the virtual scene $V E_{1}$ (see Section 4.2) is rendered at 145 frames-per-second (FPS) with the visual attention model running, as compared to 170FPS without. Detailed GPU computation times are given in Table 1. The low computation time of our visual attention model would allow it to be used in several real-time 3D applications and games.

\section{EXPERIMENTAL EVALUATION}

We conducted an experiment to evaluate the performance of our visual attention model and compare it to the state-of-the-art model of Lee et al. [6]. To the authors best knowledge, this is the only model adapted to real-time 3D exploration of VEs proposed so far.

Twelve naïve participants (10 males, 2 females) with a mean age of $31.8(\mathrm{SD}=6.4)$ participated in our experiment. They were all familiar with the first-person navigation and had normal vision.

\subsection{Experimental apparatus}

During this experiment, we used a Tobii x50 gaze tracker to compute participants' gaze position. This gaze position is considered as the ground truth. Participants were positioned in front of a 19" flat screen at a resolution of $1280 \times 1024$. The screen was $37.5 \mathrm{~cm}$ width. They were at a distance of $60 \mathrm{~cm}$ from the screen and no sound was played. The VEs were rendered in real-time with a constant refresh-rate of $75 \mathrm{~Hz}$.

The navigation in the VE was achieved using firstperson viewing mode. In this case, the virtual camera is positioned at the level of the eyes of user's avatar. We allowed three degrees of freedom of displacement: walking forward/backward and changing the horizontal and vertical camera orientation, i.e. yaw and pitch angles. Walking forward or backward was achieved using the up and down arrow keys of the keyboard. Changing camera's orientation (yaw and pitch angles) was achieved using movements of the mouse. Mouse correction and filtering were disabled. A horizontal mouse movement of $2.5 \mathrm{~cm}$ on the table resulted in a rotation of 90 degrees of the camera in the VE (36 degrees/cm). Avatar properties were inspired by real data: height was $1.75 \mathrm{~m}$ and walking speed was $1.15 \mathrm{~m} / \mathrm{s}$ [7]. 


\subsection{Procedure}

For each participant, the experiment was divided in two parts. In each of these parts, participants navigated in 3 different and randomly presented virtual environments: (1) a dynamic and textured VE with moving physical objects $\left(V E_{1}\right.$, Figure $\left.5-\mathrm{A}\right)$, a static and textured VE ( $V E_{2}$, Figure 5-B) and a static and flat colored VE ( $V E_{3}$, Figure 5-C).

During the first part, participants were asked to freely explore the virtual environment without a specific task ( $T_{f}$, use-case: virtual visits). Then, during the second part, participants were asked to search for keys hidden in the VE (task $T_{k}$ ), and to pick up a maximum of them (use-case: video games). The number of available keys was not given to participants. The second part was meant to study the performance of our model when a task is involved since the presence of task is known to have an influence on gaze patterns [10]. To take into account the task involved during the exploration, the Task $(s)$ value was set to 1.0 for surfels belonging to keys and 0.5 for all other objects. The same task value was used for the model of Lee et al. [6].

The experiment started with a training session in which participants were able to get used with the navigation protocol during 1 minute. Each navigation session of each part lasted 2 minutes. A calibration of the Tobii gaze tracker was conducted at the beginning of each part. For each participant, the overall experiment lasted 20 minutes. All sessions were recorded, and we were able to replay each session to evaluate the performances of the various visual attention models.

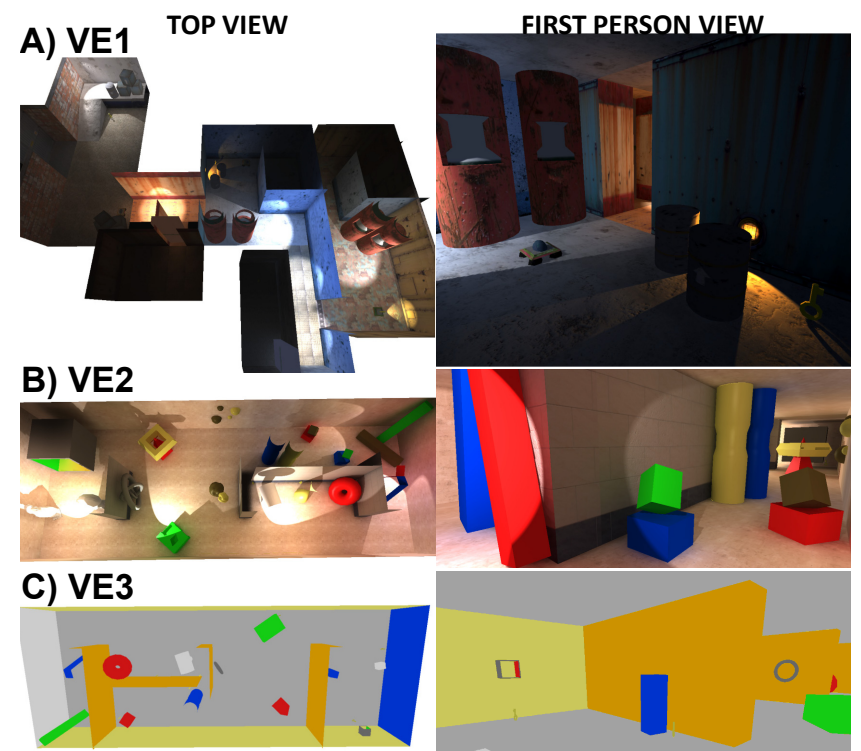

Fig. 5. The three virtual environments used in our experiment. A) Textured and dynamic VE, B) textured and static VE and C) flat colored and static VE.

\subsection{Results}

To compare our model (Mour) with the model of Lee et al. [6] (Mlee), we computed several performance indicators. Performance indicator $P_{1}$ represents the percentage of time spent by the gaze point computed by each model inside a circle area having a radius of $r$ degrees of the visual field and centered on the gaze point $G_{T}$ computed by the Tobii system (considered here as the ground truth). We have tested several $r$ values for $P_{1}: 2,4,6$ and 8 degrees (respectively corresponding to a radius of $71,143,215$ and 287 pixels). Performance indicator $P_{2}$ represents the percentage of time spent by the gaze point computed by each model on the same object as the one located at the level of $G_{T}$.

Concerning $P_{1}$, the model proposed by Lee et al. [6] was not designed to output a continuous 2D gaze point. Thus, we propose to compute the final gaze position $G_{\text {lee }}$ corresponding to the use of the model of Lee et al. [6] as the mean of positions of pixels belonging to the selected visual object (the one obtaining the highest attention). Our model is not designed to output an attended mesh. Thus, concerning $P_{2}$, we have computed the final gazed object of our model as the mesh positioned under the gaze point $G_{\text {our }}$ computed by our model.

We conducted a repeated-measures ANOVA on the dependent variable $P_{1}$ with the independent variables being the four radii $r$ and the models Mour and Mlee (Figure 6-A). The ANOVA revealed a significant main effect of the model used $(F(1,11)=313.32$, $p<0.01)$. Tukey post-hoc comparisons showed a significant difference between the two models for each radius value $r(p<0.01)$. The ANOVA also revealed a significant radius $\times$ model interaction $(F(3,33)=$ 234.68, $p<0.01)$ meaning that the difference in accuracy between the two models significantly increases when $r$ increases. The detailed comparisons of both models concerning $P_{1}$ are presented in Figure 6-B for the case where $r=4$ (which was related work [7]). In this case, we conducted a 2 (model) $\times 3$ (VEs) $\times 2$ (task) repeated-measures ANOVA. It revealed a significant main effect of the model used on performance $(F(1,11)=225.96, p<0.01)$. Then, Tukey post-hoc comparisons showed that the performance of Mour model was significantly higher than Mlee $(p<0.01)$ for all combinations of VE and Task. This is confirmed by the fact that $G_{\text {our }}$ was found to be closer to $G_{T}$ than $G_{l e e} 71 \%$ of the time. Furthermore, Tukey post-hoc comparisons revealed that neither Mour nor Mlee performance were influenced by the VE. Also, for each VE, they revealed a significant difference of performance for Mour and Mlee between $T_{f}$ and $T_{k}$ (in each case, $p<0.01$ ).

Concerning the second performance indicator $P_{2}$, a 2 (model) $\times 3$ (VEs) $\times 2$ (task) repeated-measures ANOVA again revealed a significant main effect of the 

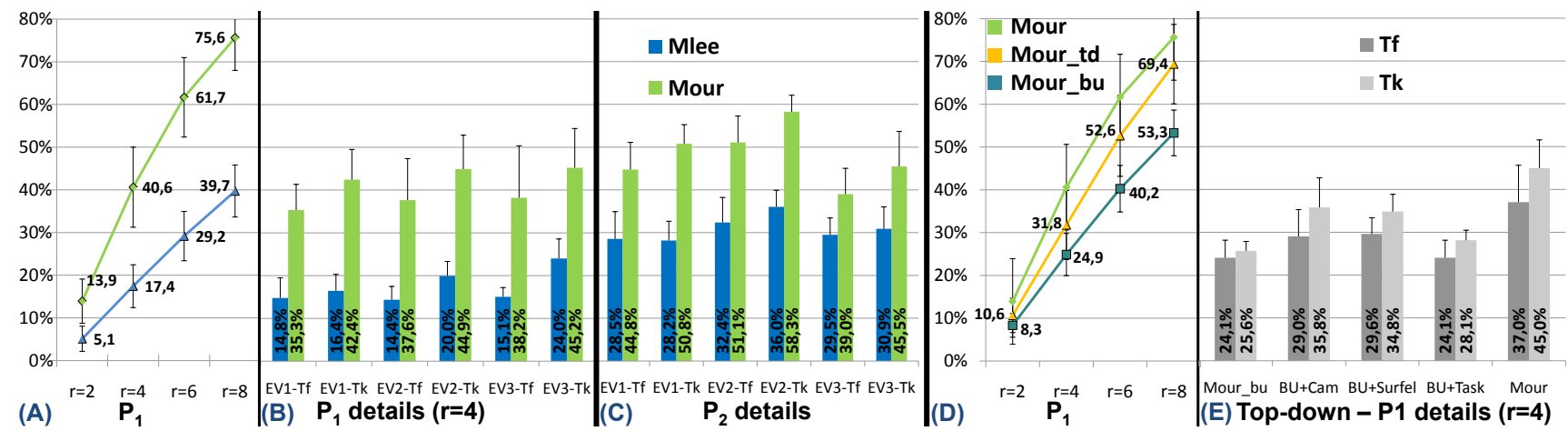

Fig. 6. Experimental results: performance obtained by various attention models (mean and standard deviation). $A, B$ and $C$ compare our visual attention model (Mour) to the existing model of Lee (Mlee). D and $E$ compare our complete model to its separated bottom-up (Mour_bu) and top-down (Mour_td) components. A, B, D and E represent the percentage of time spent by the computed gaze point in a circle having a radius of $r$ degree of the visual field centered on the ground truth gaze position. $C$ represents the percentage of time spent by the computed gaze point on the same object as the one corresponding to the ground truth gaze position. $A$ and $D$ give global performance for $r=\{2,4,6,8\}$. B, C and $\mathrm{E}$ give performance details with $r=4$ for each VE $(1,2$ and 3) and each Task $\left(T_{f}\right.$ and $\left.T_{k}\right)$.

model used on performance $(F(1,11)=560, p<0.01)$. Figure 6-C exhibits a mean accuracy of $48.2 \%$ (SD = 8.41) for our model Mour, and a mean accuracy of $30.9 \%(\mathrm{SD}=5.6)$ for the previous model Mlee. The ANOVA also revealed a significant Model $\times$ Task interaction $(F(1,11)=8.32, p<0.05)$. Tukey posthoc comparisons confirmed that Mour is significantly influenced by the task $(p<0.05)$ with an accuracy of $51,5 \%(\mathrm{SD}=7.7)$ for task $T_{f}$ and of $44.9 \%(\mathrm{SD}=7.8)$ for task $T_{k}$. The other model Mlee was not found to be significantly influenced by the task $(p=0.87)$, which leads to an accuracy for $P_{2}$ of $31,7 \%(\mathrm{SD}=5.5)$ for the task $T_{f}$ and of $30.1 \%(\mathrm{SD}=5.1)$ for $T_{k}$.

Secondly, we have compared the performance $P_{1}$ when using our complete model Mour with the use of only its bottom-up component Mour ${ }_{b u}$, or only its top-down component Mour $_{t d}$ (Figure 6-D). The ANOVA revealed a significant main effect of the model used on performance $(F(2,22)=141.75$, $p<0.01)$. Then, Tukey post-hoc comparisons showed significant differences between each model for each value of $r$ ( $p<0.01$ in each case).

The top-down part of our model has been designed around three major components: (1) the novel surfelbased representation of visual objects, (2) screen-space weights and (3) task. They correspond to Surfel part, Cam part and Task in Figure 1. We have further evaluated the elementary contribution of these three topdown components with that of the bottom-up model only by successively adding their contribution when $r=4$ (see Figure 6-E). A 5 (model) $\times 2$ (task) repeatedmeasures ANOVA revealed a significant main effect of the model used $(F(4,44)=92.73, p<0.01)$. Tukey post-hoc comparisons showed that each model was significantly different from the others excepted Mour $_{b u}+$ Cam as compared to Mour ${ }_{b u}+$ Surfel $(p=$
0.99), and Mour $b u$ as compared to Mour $b u+$ Task $(p=0.98)$. The ANOVA also revealed a significant model $\times$ task interaction $(F(4,44)=5.50, p<0.01)$. In this case, Tukey post-hoc comparisons showed that each model was significantly influenced by the task excepted Mour $_{b u}(p=0.79)$ and Mour $_{b u}+$ Task $(p=0.09)$.

\subsection{Discussion}

Overall, the results on two performance indicators show that our model performed significantly better than the previous model of Lee et al. [6] when exploring various $3 \mathrm{D}$ VE.

Firstly, concerning performance indicator $P_{1}$, our model performed significantly better than Mlee [6] (more than 100\% increase in performance), corresponding to a higher percentage of time spent by our computed gaze point close to the ground-truth gaze position (position given by the real gaze-tracker). Interestingly the performance of our model was not significantly influenced by the VE used. This suggests that our model is general enough to support many different kinds of 3D VE. Secondly, we found that both models performed significantly higher when the searching task was given to participants. This suggests that, when an implicit task is involved during the navigation, users' attention seems more predictable thanks to a higher correlation with the top-down component controlling overall gaze direction [5] and including a task related weight. It also confirms Sundstedt et al. [13] findings, suggesting that implementing a top-down component is highly beneficial for a visual attention model.

The lower accuracy of Mlee concerning $P_{1}$ is probably due to the fact that this model was designed to output a 3D object and not a continuous 2D gaze 
point on the screen. Thus, we have also compared both models using a second performance indicator $P_{2}$ which represents the percentage of time spent by the computed gaze point on the same object as the one corresponding to the ground truth gaze position. Surprisingly, even in this case, our model gives significantly better results than the previous model Mlee. Besides, performance of Mlee was actually lower than the one reported in their paper [6]. This could be due to the fact that in [6] all frames showing only the background were excluded from the analysis, whereas, in our case, all frames were kept except those were $G_{T}$ was reported as invalid $(0.98 \%)$.

Our results also revealed that the performance of our complete model was significantly higher than that when using only its bottom-up components alone or only its top-down components (Mour vs Mour $r_{b u}$ or Mour $_{t d}$ ). This suggests that visual attention models based only on a bottom-up or a top-down components would not be as effective at computing human attention as compared to using both components together. In other words, this confirms again the benefit of adding a top-down component to a visual attention model as stated in [13].

Furthermore, we have studied the contribution of separated top-down weights (Figure 6-E). Our analysis revealed that adding screen-space weights, surfel weights or task weights to the single bottom-up component Mour $_{b u}$ resulted in a significant increase in the overall performance (for both $T_{f}$ and $T_{k}$ navigations). This suggests that the component Cam, Surfel and Task are reliable top-down weights. Finally, when combining all top-down components together, the performance was also found to be significantly better, suggesting that it is important to mix several topdown components adapted to the context in which the visual attention model is used in order to correctly identify areas of interest to the user.

When replaying sessions, we could visually observe that the habituation simulation was very useful to predict participants' gaze when discovering new rooms or looking behind objects. The habituation simulation seems particularly effective during the searching task $T_{k}$. Indeed, in this case, participants were actively searching for keys in places they did not explore before. When navigating freely, the two statistical top-down components, CamRot and $\operatorname{ScrC}$, were also found helpful to better position the computed gaze point at the center of the screen and/or in the direction of the camera rotation when turning, i.e. where humans often gaze. However, we could sometimes observe that participants were rapidly parsing several areas of the screen in less than 2 seconds. In such cases, no components were able to simulate and account for this fast gaze pattern. This suggests that our model would benefit from the implementation of a more advanced gaze behavior simulator that would accurately simulate saccade and smooth pursuit gaze patterns as well as fast scene-parsing behavior.

Taken together our results suggest that our novel visual attention model could be used in various realtime 3D applications involving first-person navigation. Using a visual attention model to compute a gaze point position seems a valid approach to be used as in combination [20] or as a replacement to expensive gaze tracking systems.

\section{APPLICATION OF VISUAL ATTENTION MODEL FOR GAZE-BASED RENDERING}

In this section, we present several methods to modify the visual feedback to the user according to where he/she is looking at on the screen. We first propose a level-of-detail approach for visual effects that heavily rely on multiple texture fetches. The goal of the presented approach is to accelerate the rendering process of this type of visual effects. Then, we expose three different gaze-based visual effects inspired from the Human visual system which are adapted in real-time to the user's gaze position. The goal when using these effect is to improve user's perception and immersion in a VE.

\subsection{Gaze-based per-pixel level-of-detail}

\subsubsection{Concept}

In the rendering research area, the gaze point has been mainly used to manage the level-of-detail of the rendered VE in order to accelerate the rendering process and thus maintains a high refresh rate of the application. First, it has been used to reduce the details of objects that are far away from the point of interest of the user on the screen. This has been achieved either by progressively decimating meshes [1] or by choosing an appropriate mesh to render from a predefined set [6]. Considering volume rendering, the distance from the gaze point has been used to fetch appropriate mipmap levels from the volumetric texture along each ray cast inside the volume when computing the lighting solution [21]. A similar process could be used to tune the quality of off-line rendering methods per pixel [8].

Nowadays, texture sampling on graphic hardware become more and more costly compared to Arithmetic Logic Unit operations. Unfortunately, a lot of visual effects rely on multiple texture sampling for each pixel of the rendered image. Such effects are percentage closer soft shadow mapping, relief mapping or sub-surface light scattering. In this Section, we propose to dynamically adapt the quality of the Colored Stochastic ShadowMapping (CSSM) method [22] by exploiting the gaze position of the user on the screen. To reduce the computation time, we propose to dynamically tune the number of texture samples required for each pixel based on their distance to the user's gaze point. For pixels close to the gaze position, a lot of samples will 
be taken in order to ensure an accurate visual quality. Then, the farther the pixels from the gaze position, the less samples of samples.

\subsubsection{Gaze-base colored stochastic shadow mapping}

The colored stochastic shadow mapping (CSSM) algorithm solves an important challenge of real-time rendering: order-independent and colored shadows. It means that the rendering of partially covered and colored transmissive surfaces can be properly rendered in a unified way. This method uses a stochastic approach to sample the attenuation of light along a ray and distribute the sample spatially in the shadow map. For more details about this method, we refer the reader to the original paper [22]. As for all stochastic approaches, the drawback of this method is that it requires a large sampling kernel when rendering the shadow map to avoid high frequency noise. This is especially true in the case of CSSM for surfaces having low transparency. The authors have proposed a box-plus-cross-shaped filter kernel consisting of 13 samples which results in low noise for a reasonably wide type of transparent surfaces. However, for low transparency surfaces that block most of the light, a lot of noise can appear (Figure 7-A). This is especially true when light rays travel through several surfaces. To solve this issue, the straightforward approach is to densely sample a wider area of the shadow map (CSSMHQ). As visible on Figure 7-B, using a 11-pixel wide box filter mostly removes the noise and results in a smooth color. However, this filter requires 121 samples per pixel. As a consequence, the refresh rate of the application drops significantly (see Table 2).

\begin{tabular}{|c|c|c|}
\hline Algorithm & $512 \times 512$ & $2048 \times 2048$ \\
\hline CSSM & $2.7 \mathrm{~ms}(235 \mathrm{fps})$ & $4.6 \mathrm{~ms}(113 \mathrm{fps})$ \\
\hline CSSMHQ & $19.4 \mathrm{~ms}(44 \mathrm{fps})$ & $24.9 \mathrm{~ms}(30 \mathrm{fps})$ \\
\hline CSSMGAZE & $5.6 \mathrm{~ms}(130 \mathrm{fps})$ & $7.6 \mathrm{~ms}(80 \mathrm{fps})$ \\
\hline
\end{tabular}

TABLE 2

Performance of the original CSSM algorithm as compared to the CSSMHQ (11 pixel wide filter) and CSSMGAZE (dynamic box filter width) approaches for two resolutions of the shadow map. Performances are given in milliseconds and frames-per-second when rendering the scene depicted on Figure 7.

To improve the CSSM algorithm, we propose to dynamically modify the number of samples taken per pixel based on their distance to the gaze point position (CSSMGAZE). To this aim, we define a quality value $q$ for each pixel which is 1 at the gaze position and that linearly drops to 0 at a distance of half the height of the display screen. The width $w$ of the box filter is then set per pixel to $w=1+2 \times\left\lfloor q \times w_{\max }\right\rfloor$. After preliminary testing, we have set $w_{\max }=6$ to get the high quality box filter of 11-pixel wide around the gaze position.
Using the CSSMGAZE algorithm, the lighting solution near the gaze position is of high quality (Figure 7 ). Then, the filtering quality progressively decreases when moving away from the gaze position (Figure 7). As the user is not looking at this outer area, he/she is not able to perceive the difference while the application is able to render at a higher refresh-rate compared to the CSSMHQ algorithm. The computation time and effect of the dynamic box filter are shown on Table 2.

\subsubsection{Conclusion}

Dynamically modifying the sampling kernel of visual effects on today's hardware seems a valid approach. This may be due to the fact that dynamic branching in shader is becoming increasingly effective. We believe that other visual effects such as relief mapping or percentage closer soft shadow mapping could also greatly benefit in using such a dynamic scaling of the number of texture fetches. It is also important to note that the cost of using our visual attention model to predict the user's attention is easily amortized when using such a method considering the ratio between the gain in visual quality and the reduction in computation time.

\subsection{Gaze-based visual effects}

The gaze point position can also be used to improve the visual feedback to the user by adding gaze-based visual effects. Such effects are designed to improve the perception and immersion of the users in VE. To this aim, we present three visual effects inspired from the human visual system that take into account the user's gaze point position on the display peripheral. We present two effects we have already proposed in a previous work (compensated camera motion and depth-of-field blur [2]) as well as a novel one (luminance adaptation).

\subsubsection{Gaze-based compensated camera motion}

When moving in a virtual environment, it is important that users feel like they are effectively walking. This is important to get a much higher immersion feeling. Typically, when walking in the virtual environment, the camera features a sinusoidal motion simulating the movement of the head when walking [2]. Thus, the user have the impression of walking. However, the visual flow resulting from this motion can be very high and thus can become annoying. This perturbed visual flow can make the fixation of a single point in the VE very hard for the user.

To overcome this issue, we propose to apply a compensation to the camera motion in order to smooth the visual flow at the level of the user's gaze point [2]. This more complex camera motion simulates the vestibulo-ocular reflexes of our eyes which allow a human to fixate a single point in space while he 

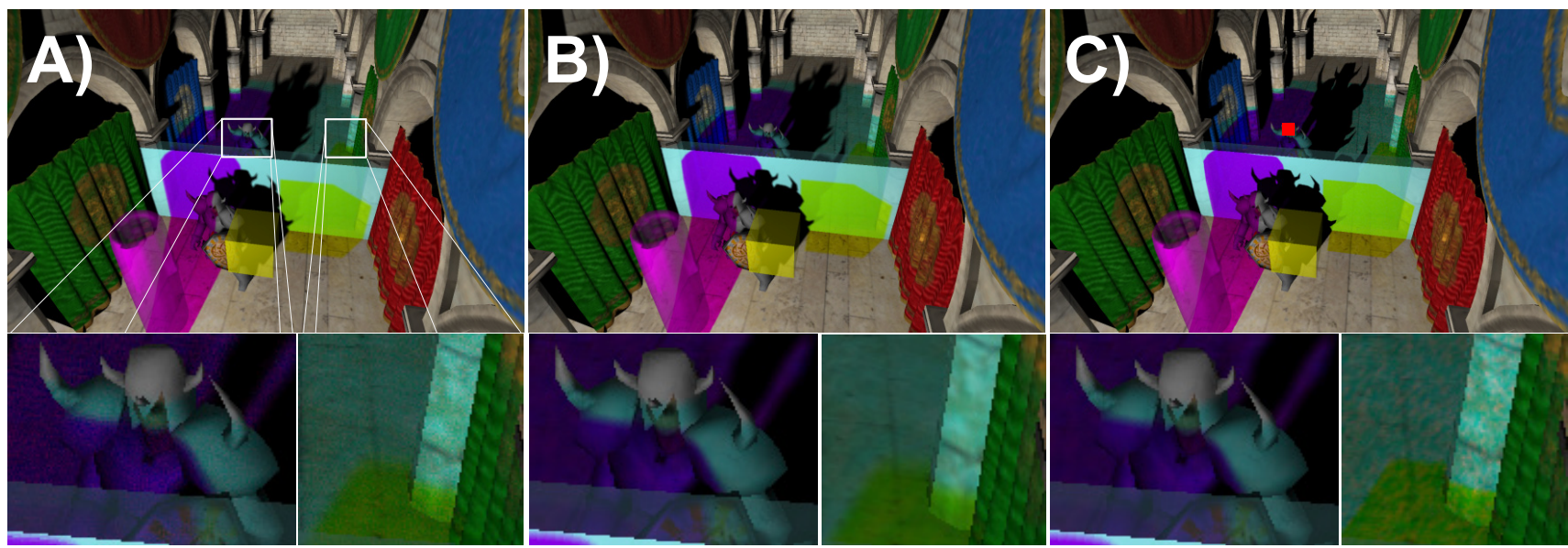

Fig. 7. Rendering of the Crytek's Sponza Atrium model [23] with transparent surfaces using the original CSSM algorithm (A), the CSSMHQ using 11-pixels wide filter (B) or the CSSMGAZE using dynamic box filter width (C) approaches. Close-up views show the difference in quality of the different algorithm. The red square in image $\mathrm{C}$ represents the gaze position of the user.

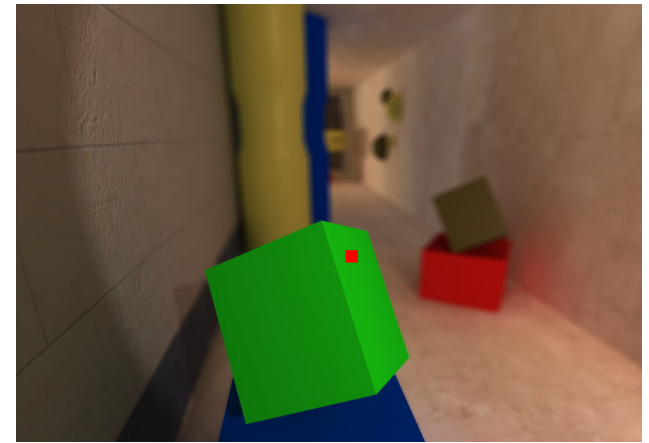

Fig. 8. Depth-of-field blur dynamically adapted to user's gaze point position (small red square) as estimated by a real-time visual attention model.

is walking by compensating his/her body and head motion. As a consequence, the user still has the impression of walking in the VE while being now able to fixate a single point in space without any effort.

Previous work has revealed that participants significantly preferred the camera motion when it was compensated and adapted to where they were looking at on the screen [2]. Furthermore, Terziman et al. [24] have presented some results suggesting that a compensated camera motion could help to better perceive traveled distances when walking in a VE.

\subsubsection{Gaze-based depth-of-field blur}

In reality, we do not perceive our whole surrounding environment sharply but partially blurred. This is mostly due to the fact that the chrystalin in our eyes act as a lens: objects we are looking at, i.e. at the focal distance, appear sharp. Other objects in the visual field appear progressively blurred according to their distance to the focal distance.

In previous work [2], we have proposed to adapt a depth-of-field (DoF) blur effect to user's gaze point position on the visual display. To this aim, we dynamically adapt the focal distance to the distance at which the user is looking at in the VE from the virtual camera. Then, a blurred version of the original sharp image can be computed using a lens equation [2] (Figure 8).

Previous work has revealed that participants significantly preferred the depth-of-field blur when it was dynamically adapted to where they were looking at [2]. Furthermore, Moehring et al. [25] have shown that such a blur effect could effectively help users to better reproduce distances in VE.

\subsubsection{Gaze-based luminance adaptation}

High dynamic range (HDR) images refer to picture being composed of color values not limited to a maximum value of 1.0. Displaying such images on low dynamic range (LDR) display peripherals is not a trivial task and is still a very active research field in the graphics community. The goal of a tone-mapping operator is to make all the parts of a HDR image visible to the viewer when displayed on a LDR screen [26]. The human visual system is able to perceive a wide range of luminance values from dim interiors to outside areas brightly lit by the sun [26] at the same time. It is also able to adapt itself dynamically when the lighting condition changes rapidly. Inspired by this capacity of the human visual system, we propose a gazed-based tone-mapping algorithm.

The method we propose is targeted toward realtime 3D applications featuring high dynamic range lighting. To this aim, it is based on the tone-mapping method proposed by Reinhard et al. [26]. This tonemapping method computes the final luminance $L_{f}$ of each pixel from the original luminance value $L$ using equation 9 where $g$ is middle grey, $L_{\min }$ is the minimum luminance to be mapped to black, $L_{\text {white }}$ 

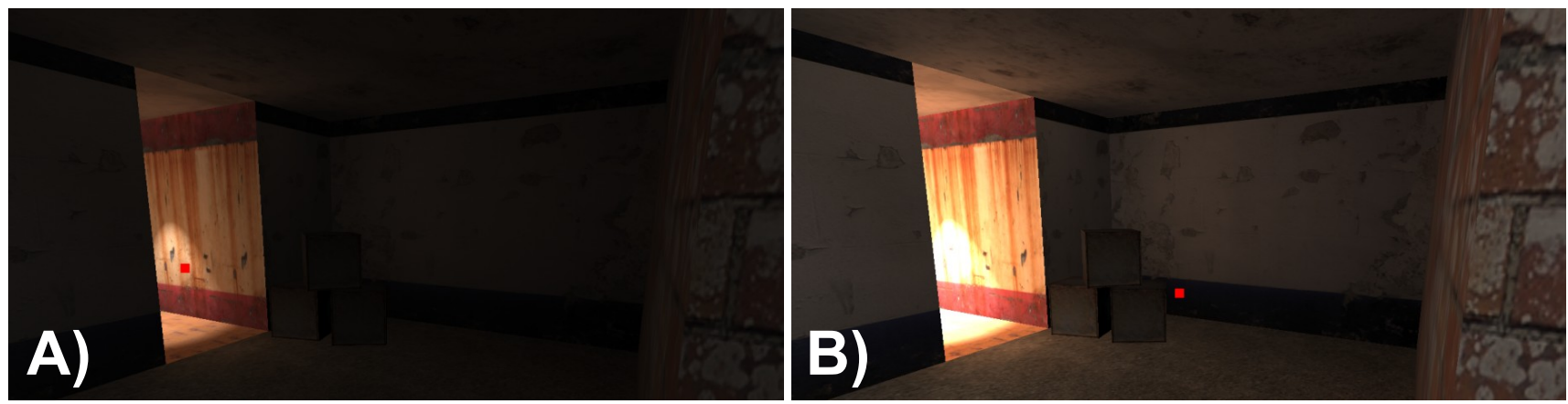

Fig. 9. Dynamic luminance adaptation based on the user's gaze point on the screen (the red square).

the smallest luminance that will be mapped to white and $L_{\text {mean }}$ the mean luminance of the view field. To compute the value of these parameters, we consider a low resolution version of the displayed HDR image. For the sake of real-time performance, after preliminary testing, we propose to use the level of its mipmap pyramid having a width of 128 . From this texture, we can easily compute $L_{\min }$ as the minimum luminance and $L_{\text {white }}$ as the maximum luminance of all the pixels. The $L_{\text {mean }}$ value is supposed to represent the mean luminance of the whole scene image. In our case, we choose to set this value to the luminance that is at the position the user is looking at, using the bilinear filtered luminance value from the four neighboring pixels.

$$
\begin{aligned}
& \left.L=g \frac{\left(L-L_{\text {min }}\right.}{L_{\text {mean }}}\right) \\
& L_{f}=\frac{L\left(1+\frac{L}{L_{\text {white }}}\right)}{1+L}
\end{aligned}
$$

This dynamic adaptation of the Reinhard tonemapping operator allows [26] the user to feel like he is looking at a HDR environment, although he can't be dazzled, thanks to the fact that the synthesized image display on the LDR display peripheral is constantly adapted to the area he is looking at. The effect can be smoothed by applying a low pass filter on the $L_{\text {mean }}$ to simulate temporal adaptation. The result of using this method is visible on Figure 9. You can notice that when the user is looking at a bright region, the overall image appears dimmer in order to perceive more detail where the user is looking at. Conversely, the scene appears brighter when the user is looking at dark areas.

As with the compensated camera motion and depth-of-field blur, the expected outcomes for the users are a higher immersion feeling and a better perception of the VE he is exploring. Although not being a perceptually correct simulation of the human visual system, we believe that using such visual effects inspired from it is the way to follow in order to improve the overall experience of users exploring a virtual world.

\section{Conclusion}

In this paper, we have presented a novel visual attention model to compute user's gaze position on screen in real-time. This model is specifically designed for the exploration of 3D virtual environments and can compute, for the first time, a continuous gaze point position. This novel visual attention model is made of two components: a bottom-up and a top-down component. Contrary to previous models, which used a mesh-based representation of visual objects, we have introduced a new data representation based on surfels. We propose this solution to close the gap between screen-space and object-space approaches. The bottom-up and top-down components are combined to create a final attention map and compute the continuous gaze point.

We conducted an experiment to study the performance of our method. Overall, the results show that our model performed significantly better than a state-of-the-art model when exploring various 3D virtual environments with an increase in performance of more than $100 \%$. Taken together our results suggest that our novel visual attention model could be used in various real-time applications such as video games or virtual reality systems.

Finally, we have proposed to take advantage of the computed gaze point to adapt the rendering of the virtual environment to the user's attention. To this aim, we have first proposed to dynamically adapt the quality of visual effects that heavily rely on texture fetching, a costly operation on today's hardware. We have shown that it is possible to dynamically adapt per-pixel the sampling kernel of the filtering kernel according to user's gaze position. This enables the acceleration of the rendering process in order to maintain a high frame-rate. Second, we have exposed several gaze-based visual effects inspired from the human visual system and adapted to the user's visual attention in the virtual scene. This effects aim at improving the perception and immersion of the user.

\section{FUTURE WORK AND PERSPECTIVES}

Further research efforts could first be directed toward the improvement of our visual attention model. We 
would like to study and propose a better way to combine the several components used in our model since a linear combination may be not the best way to go. Furthermore, the gaze pattern simulator could be enhanced by simulating more advanced gaze patterns such as fixation/saccade or smooth pursuit. Finally, we would like to further evaluate our model in various VEs and contexts such as virtual training, architectural visits or games.

Concerning the gaze-based visual effects, it would be interesting to improve the luminance adaptation method in order to not only take into account the point the user is looking at, but also the whole environment surrounding the user. This could be particularly useful when rendering images on a low dynamic range CAVE-like display peripheral. More effects could also be researched such as a stereo rendering algorithm that would take into account user's gaze position in order to reduce disparity and compute a better convergence point. This could make this technology more appealing to users. We would also like to conduct experiments in order to study user's subjective preferences toward these effects as well as their effects on the perception of the virtual environments.

After gesture based interaction, e.g. the WiiMote or Kinect, we believe that computing the user's gaze could represent the next big step in human-computer interaction for daily use. It could be achieved using either a gaze-tracking system, a visual attention model or even a combination of both [27]. Many 3D applications could benefit from being aware of the users attention. Nowadays, game developers are increasingly interested in emerging technologies to study gamers reactions to their games during playtests [28]. For instance, monitoring gamers physiological activities and states could be used to evaluate their state of mind and level of stress in order to adapt the difficulty of the game. We strongly believe that this feature could define a totally new area in the field of application/game design researches such as for novel attention-based visual effects, gaze-based interaction protocols or gaze-based gameplay.

\section{REFERENCES}

[1] D. P. Luebke and B. Hallen, "Perceptually-driven simplification for interactive rendering," in Proceedings of the 12th Eurographics Workshop on Rendering Techniques, 2001, pp. 223234.

[2] S. Hillaire, A. Lécuyer, R. Cozot, and G. Casiez, "Using an eye-tracking system to improve camera motions and depth-offield blur effects in virtual environments," Proc. of IEEE Virtual Reality, pp. 47-50, 2008.

[3] A. Glenstrup and T. Engell-Nielsen, "Eye controlled media : Present and future state," Master thesis, University of Copenhaguen, 1995.

[4] L. Itti, C. Koch, and E. Niebur, "A model of saliency-based visual attention for rapid scene analysis," in IEEE Transactions on Pattern Analysis and Machine Intelligence, vol. 20, no. 11, pp. 1254-1259, 1998.
[5] L. Itti, "Quantifying the contribution of low-level saliency to human eye movements in dynamic scenes," In Visual Cognition, vol. 12, pp. 1093-1123, 2005.

[6] S. Lee, G. Kim, and S. Choi, "Real-time tracking of visually attended objects in virtual environments and its application to LOD," In IEEE Transactions on Visualization and Computer Graphics, vol. 15, no. 1, pp. 6-19, Jan.-Feb. 2009.

[7] S. Hillaire, A. Lécuyer, G. Breton, and T. Regia-Corte, "Gaze behavior and visual attention model when turning in virtual environments," in Proceedings of ACM Symposium on Virtual Reality Software and Technology, 2009, pp. 43-50.

[8] K. Cater, A. Chalmers, and G. Ward, "Detail to attention: exploiting visual tasks for selective rendering," Proc. of the 14th Eurographics workshop on Rendering, pp. 270-280, 2003.

[9] A. M. Treisman and G. Gelade, "A feature-integration theory of attention." In Cognitive Psychology, vol. 12, no. 1, pp. 97-136, 1980.

[10] D. Yarbus, Eye motion and vision. Plenum Press, 1967.

[11] P. Longhurst, K. Debattista, and A. Chalmers, "A GPU based saliency map for high-fidelity selective rendering," Proc. of the 4th international conference on Computer graphics, virtual reality, visualisation and interaction in Africa, pp. 21-29, 2006.

[12] V. Navalpakkam and L. Itti, "Modeling the influence of task on attention," In Vision Research, vol. 45, no. 2, pp. 205-231, 2005.

[13] V. Sundstedt, E. Stavrakis, M. Wimmer, and E. Reinhard, "A psychophysical study of fixation behavior in a computer game," in Proceedings of the 5th symposium on Applied perception in graphics and visualization, 2008, pp. 43-50.

[14] C. Sears and Z. Pylyshyn, "Multiple object tracking and attentional processing," Journal of Experimental Psychology, vol. 54, no. 1 , pp. $1-14,2000$.

[15] A. R. Robertson, "Historical development of cie recommended color difference equations," In Color Research and Application, vol. 15, no. 3, pp. 167-170, 1990.

[16] H. Chen and X. Liu, "Lighting and material of halo 3," Siggraph 2008 courses, 2008.

[17] L. Williams, "Casting curved shadows on curved surfaces," Proc. of ACM SIGGRAPH, vol. 12, pp. 270-274, 1978.

[18] S. Hillaire, A. Lécuyer, R. Cozot, and G. Casiez, "Depth-offield blur effects for first-person navigation in virtual environments," IEEE Computer Graphics and Applications, vol. 28, no. 6, pp. 47-55, 2008.

[19] D. A. Robinson, "The mechanics of human smooth pursuit eye movement," Journal of Physiology, vol. 180, pp. 569-591, 1965.

[20] S. Hillaire, G. Breton, N. Ouarti, R. Cozot, and A. Lcuyer, "Using a visual attention model to improve gaze tracking systems in interactive 3d applications," Computer Graphics Forum, vol. 29, no. 6, pp. 1830-1841, 2010.

[21] M. Levoy and R. Whitaker, "Gaze-directed volume rendering," in Proceedings of the ACM symposium on Interactive 3D graphics, 1990, pp. 217-223

[22] M. McGuire and E. Enderton, "Colored stochastic shadow maps," Proceedings of the ACM Symposium on Interactive $3 D$ Graphics and Games, 2011. [Online]. Available: http://research.nvidia.com/publication/coloredstochastic-shadow-maps

[23] F. Meinl, "Crytek sponza atrium," http://www.crytek.com/cryengine/cryengine3/downloads, 2010.

[24] L. Terziman, A. Lécuyer, S. Hillaire, and J. Wiener, “Can camera motions improve the perception of traveled distance in virtual environments?" Proc. of IEEE Virtual Reality, p. 131134, 2009.

[25] M. Moehring, A. Gloystein, and R. Doerner, "Issues with virtual space perception within reaching distance: Mitigating adverse effects on applications using hmds in the automotive industry," in Proceedings of the 2009 IEEE Virtual Reality Conference, 2009, pp. 223-226.

[26] E. Reinhard, M. Stark, P. Shirley, and J. Ferwerda, "Photographic tone reproduction for digital images," in Proceedings of the 29th annual conference on Computer graphics and interactive techniques, 2002, pp. 267-276.

[27] S. Hillaire, G. Breton, N. Ouarti, R. Cozot, and A. Lécuyer, "Using a visual attention model to improve gaze tracking systems in interactive 3d applications," Computer Graphics Forum, vol. 29, pp. 47-55, 2010. 
[28] M. Ambinder, "Valve's approach to playtesting: The application of empiricism," in Game Developer's Conference, 2009. 